\title{
ON DUNFORD-PETTIS OPERATORS THAT ARE PETTIS-REPRESENTABLE
}

\author{
ELIAS SAAB
}

\begin{abstract}
Let $E$ be a Banach space. It is shown that if every DunfordPettis operator $T: L^{1}[0,1] \rightarrow E^{*}$ is Pettis-representable, then every operator $T: L^{1}[0,1] \rightarrow E^{*}$ is Pettis-representable.
\end{abstract}

It is well known that a bounded linear operator $T$ from $L^{1}[0,1]$ into a Banach space $E$ that is Bochner or Pettis-representable is a Dunford-Pettis operator, i.e., $T$ maps weakly convergent sequences into norm convergent sequences. It was suspected for a while that any Dunford-Pettis operator $T: L^{1}[0,1] \rightarrow L^{1}[0,1]$ is Bochner representable, but Costé (see [3, p. 90]) gave an example of a convolution type operator $T: L^{1}[0,1] \rightarrow L^{1}[0,1]$ that is a Dunford-Pettis operator but is not Bochner representable. Costé's example suggested the following problem: If every Dunford-Pettis operator from $L^{1}[0,1]$ into a Banach space $E$ is Bochner representable, is every bounded linear operator $T: L^{1}[0,1] \rightarrow E$ Bochner representable? In [2] Bourgain solved the above problem affirmatively. A parallel problem to the one solved by Bourgain can now be asked as follows: If every DunfordPettis operator $T$ from $L^{1}[0,1]$ to a Banach space $E$ is Pettis-representable, is every bounded linear operator $T: L^{1}[0,1] \rightarrow E$ Pettis-representable?

In [10] we showed that the answer to the above problem is positive when the Banach space $E$ is complemented in a Banach lattice. In fact under this hypothesis one can conclude that every bounded linear operator $T: L^{1}[0,1] \rightarrow E$ is Bochner representable (see [5]).

In this paper, we will show that the answer is also positive for dual Banach spaces, indeed we shall show that if every Dunford-Pettis operator $T$ from $L^{1}$ into a dual Banach space $E^{*}$ is Pettis-representable then every bounded linear operator is Pettis-representable.

By an operator between two Banach spaces, we always mean a bounded linear operator. All the notions used in this paper and not defined can be found in $[3,5]$. Let $E$ be a Banach space and let $T$ be an operator from $L^{1}[0,1]$ to $E$. The operator $T$ is said to be Bochner (resp. Pettis) representable if there exists $g:[0,1] \rightarrow E$ Bochner-integrable and essentially bounded (resp. Pettis-integrable and scalarly essentially bounded) such that for every $f$ in $L^{1}[0,1], T(f)=$ Bochner- $\int_{0}^{1} f \cdot g d \lambda$ (resp., Pettis- $\int_{0}^{1} f \cdot g d \lambda$ ).

A Banach space $E$ is said to have the Radon-Nikodym property (RNP) (resp., the weak-Radon-Nikodym property (WRNP)), if every operator $T: L^{1}[0,1] \rightarrow E$ is Bochner-representable (resp., Pettis-representable). For more about the RNP and the WRNP see $[3,5,7,8,9]$.

Received by the editors August 15, 1981.

1980 Mathematics Subject Classification. Primary 46B22, 46G10.

Key words and phrases. Dunford-Pettis operators, Pettis-representable operators.

(c) 1982 American Mathematical Society 
THEOREM 1. Let $E$ be a Banach space. If every Dunford-Pettis operator $T: L^{1}[0,1] \rightarrow E^{*}$ is Pettis-representable then $E$ does not contain any isomorphic copy of $l_{1}$.

Proof. If not, then $l_{1}$ is isomorphic to a subspace of $E$. Let $S: l^{1} \rightarrow E$ be the embedding of $l_{1}$ into $E$ and let $V$ be a quotient map from $l_{1}$ onto $C[0,1]$ the space of continuous functions on the interval $[0,1]$ and let $V^{*}=U$ be the adjoint map $U: M[0,1] \rightarrow l^{\infty}$ where $M[0,1]=C[0,1]^{*}$ the Banach space of all Radon measures on $[0,1]$.

It is clear that $U$ is an embedding. The space $M[0,1]$ is an $L$-space, therefore, by a result of Grothendieck [6] the operator $U$ has a lifting $Q$ from $M[0,1]$ to $E^{*}$ such that $S^{*} Q=U$. It is evident that $Q$ is an embedding. Let $R$ be the natural embedding of $L^{1}[0,1]$ into $M[0,1]$, and let $T$ be a Dunford-Pettis operator from $L^{1}[0,1]$ to $L^{1}[0,1]$ that is not Bochner-representable in $L^{1}[0,1][3$, p. 92]. Consider the following diagram

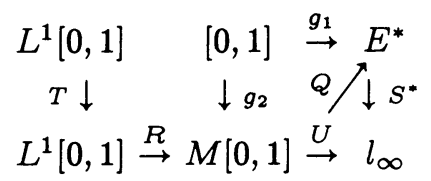

where $g_{1}$ and $g_{2}$ are to be specified shortly. The operator $Q R T: L^{1}[0,1] \rightarrow E^{*}$ is Dunford-Pettis, therefore $Q R T$ is Pettis-representable. Let $g_{1}:[0,1] \rightarrow E^{*}$ be its Pettis-derivative, that is

$$
Q R T(f)=\text { Pettis- } \int_{0}^{1} f g_{1} d \lambda \text { for every } f \in L^{1}[0,1] .
$$

Let $g_{2}$ be a weak ${ }^{*}$-derivative of the operator $R T: L^{1}[0,1] \rightarrow M[0,1]$, that is,

$$
R T(f)=\omega^{*}-\int_{0}^{1} f \cdot g_{2} d \lambda \text { for every } f \in L^{1}[0,1]
$$

and this means that $\langle\phi, R T(f)\rangle=\int_{0}^{1}\left\langle\phi, g_{2}(t)\right\rangle d \lambda$ for every $\phi$ in $C[0,1]$ [3, VI.8.6]. The function $g_{2}$ takes its values in the weak* closure of the image by $R T$ of the unit ball of $L^{1}[0,1]$. Notice that (1) implies that $S^{*} Q R T(f)=$ Pettis- $\int_{0}^{1} f S^{*} g_{1} d \lambda$ for every $f \in L^{1}[0,1]$. But $S^{*} Q=U$, hence

$$
U R T(f)=\text { Pettis- } \int_{0}^{1} f S^{*} g_{1} d \lambda \text { for every } f \in L^{1}[0,1]
$$

On the other hand (2) implies

$$
U R T(f)=\omega^{*}-\int_{0}^{1} f U g_{2} d \lambda \text { for every } f \in L_{1}[0,1]
$$

because $U$ is weak ${ }^{*}$ to weak* continuous. (3) and (4) imply that for every $a \in l_{1}$ and every Lebesgue measurable subset $A$ in $[0,1] \int_{A}\left\langle a, S^{*} g_{1}\right\rangle d \lambda=\int_{A}\left\langle a, U g_{2}\right\rangle d \lambda$. This implies that $S^{*} g_{1}=U g_{2}(t) \lambda$-almost everywhere, because $l_{1}$ is separable. The function $S^{*} g_{1}:[0,1] \rightarrow l_{\infty}$ is Pettis-integrable, therefore, the function $U g_{2}:[0,1] \rightarrow$ $l_{\infty}$ is Pettis-integrable. Hence $g_{2}:[0,1] \rightarrow M[0,1]$ is Pettis-integrable because $U$ is an embedding, hence

$$
R T(f)=\text { Pettis- } \int_{0}^{1} f \cdot g_{2} d \lambda \quad \text { for every } f \in L^{1}[0,1] .
$$


Let $P$ be the usual projection from $M[0,1]$ into $R\left(L^{1}[0,1]\right)$ and

$$
W=R^{-1}: R\left(L^{1}[0,1]\right) \rightarrow L^{1}[0,1]
$$

The map $h=W P g_{2}:[0,1] \rightarrow L_{1}[0,1]$ is weakly measurable, therefore it is strongly measurable by the Pettis measurability theorem [3, p. 42], hence it is Bochner-integrable because it is bounded. Now (5) implies that

$$
\begin{aligned}
T(f) & =W P R T(f)=\text { Pettis- } \int_{0}^{1} f W P g_{2} d \lambda \\
& =\text { Bochner- } \int_{0}^{1} f \cdot h d \lambda
\end{aligned}
$$

for every $f \in L^{1}[0,1]$. This shows that $T$ is Bochner-representable, a contradiction that finishes the proof.

Theorem 1 and the result of [1] or [7] give the following theorem.

THEOREM 2. Let $E$ be a Banach space. Then the following statements are equivalent:

(i) The space $E$ does not contain a copy of $l_{1}$;

(ii) The space $E^{*}$ has the weak Radon-Nikodym property;

(iii) Every operator $T: L^{1}[0,1] \rightarrow E^{*}$ is Pettis-representable;

(iv) Every Dunford-Pettis operator $T: L^{1}[0,1] \rightarrow E^{*}$ is Pettis-representable.

Proof. (i) $\rightarrow$ (ii) is due to Bourgain [1] and Janicka [7].

(ii) $\rightarrow$ (iii) $\rightarrow$ (iv) are evident.

(iv) $\rightarrow$ (i) is Theorem 1 .

It is worth mentioning the well-known fact that (i), (ii) or (iii) of Theorem 2 is equivalent to the following statement: (v) Every operator $T: L^{1}[0,1] \rightarrow E^{*}$ is Dunford-Pettis [9].

\section{REFERENCES}

1. J. Bourgain, On martingales in conjugate Banach spaces (unpublished).

2. , Dunford-Pettis operators on $L^{1}$ and the Radon-Nikodym property, Israel J. Math. 37 (1980), 34-47.

3. J. Diestel and J. J. Uhl, Jr., Vector measures, Math. Surveys, no. 15, Amer. Math. Soc., Providence, R. I., 1977.

4. D. G. Fremlin and M. Talagrand, A decomposition theorem for additive set-functions, with applications to Pettis integrals and ergodic means, Math. Z. 168 (1979), 117-242.

5. N. Ghoussoub and E. Saab, On the weak Radon-Nikodym property, Proc. Amer. Math. Soc. 81 (1981), 81-84.

6. A. Grothendieck, Une caractérisation vectorielle métrique des espaces $L^{1}$, Canad. J. Math. 7 (1953), 552-561.

7. L. Janicka, Wlasnosci typu Radona-Nikodyma dla przestrzeni Banacha, Thesis, Wrocław, Poland, 1978.

8. K. Musial, The weak Radon-Nikodym property in Banach spaces, Studia Math. 64 (1978), 151174.

9. L. H. Riddle and J. J. Uhl, Jr., Martingales and the fine line between Asplund spaces and spaces not containing a copy of $l_{1}$ (preprint).

10. E. Saab, On Dunford-Pettis operators, Canad. Math. Bull. (to appear). 65211

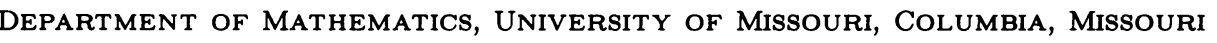

